\title{
ACÚMULO DE NITROGÊNIO, FÓSFORO E POTÁSSIO PELO ALGODOEIRO SOB IRRIGAÇÃO CULTIVADO EM SISTEMAS CONVENCIONAL E ADENSADO(1)
}

\author{
Ciro Antônio Rosolem(2), Fábio Rafael Echer(2), Izaías Pinheiro \\ Lisboa $^{(2)}$ \& Talita Silva Barbosa(2)
}

\begin{abstract}
RESUMO
O adensamento da cultura do algodão aumenta a competição entre plantas por recursos, como luz, nutrientes e água; logo, o período de florescimento é reduzido e, consequentemente, a marcha de acúmulo de matéria seca é alterada. $\mathrm{O}$ objetivo deste trabalho foi determinar a marcha de absorção de $\mathrm{N}$, $\mathrm{P}$ e K pelo algodoeiro, identificando a época de maior absorção e a quantidade absorvida e exportada em espaçamentos adensado $\left(48 \mathrm{~cm}-20,58\right.$ plantas $\left.\mathrm{m}^{-2}\right)$, intermediário $\left(75 \mathrm{~cm}-13,30\right.$ plantas $\left.\mathrm{m}^{-2}\right)$ e convencional $\left(96 \mathrm{~cm}-10,39\right.$ plantas $\left.\mathrm{m}^{-2}\right)$. Foram amostradas três plantas úteis por parcela aos 46, 69, 99, 139, 148 e 166 dias após a emergência (DAE), em que foram determinados o acúmulo de matéria seca, $\mathrm{N}, \mathrm{P}$ e K. O acúmulo de matéria seca nas plantas cultivadas no espaçamento de $48 \mathrm{~cm}$ foi maior no início do desenvolvimento, igualando-se às demais ao final do ciclo, o que resultou em maior acúmulo de N entre os 69 e 99 DAE, no menor espaçamento. $\mathrm{O}$ adensamento antecipa o pico de absorção de nutrientes, sugerindo a antecipação das adubações de cobertura com $\mathrm{N}$ e $\mathrm{K}$ no algodoeiro cultivado nesse sistema. Para condições de fertilidade média/alta, a dose de nutrientes a ser aplicada não precisaria ser alterada em função do aumento da densidade de plantas, pois não há variação nas quantidades de N, P e K exportados por kg de algodão produzido, em fibra ou em caroço.
\end{abstract}

Termos de indexação: Gossypium hirsutum, acúmulo de nutrientes, população de plantas, nutrição mineral.

(1) Recebido para publicação em 17 de março de 2011 e aprovado em 30 de novembro de 2011.

(2) Universidade Estadual Paulista, Faculdade de Ciências Agronômicas, Campus de Botucatu, Caixa Postal 237, CEP 18603-970 Botucatu (SP). E-mail: rosolem@fca.unesp.br fabioecher@fca.unesp.br, iplisboa@fca.unesp.br, e tsbarbosa@fca.unesp.br 


\title{
SUMMARY: NITROGEN, PHOSPHORUS AND POTASSIUM ACCUMULA- TION IN COTTON GROWN IN CONVENTIONAL AND NARROW SPACING
}

\begin{abstract}
An increase in plant density of cotton (Gossypium hirsutum L., cv. FM $966 \mathrm{LL}$ ) results in increased interplant competition for resources, and consequently the flowering period is shortened and the rate of dry matter accumulation changed. The purpose of this study was to determine the $N, P$ and $K$ uptake rates by cotton plants, identifying the time of greatest absorption and the total uptake and export in narrow spacing $\left(48 \mathrm{~cm}-20.58\right.$ plants $\left.\mathrm{m}^{-2}\right)$, intermediate $\left(75 \mathrm{~cm}-13.30\right.$ plants $\left.\mathrm{m}^{-2}\right)$ and conventional spacing $\left(96 \mathrm{~cm}-10.39\right.$ plants $\left.\mathrm{m}^{-2}\right)$. Three competitive plants were sampled per plot 46, 69, 99, 139, 148, and 166 days after plant emergence, when dry matter accumulation, and $N$, $P$ and $K$ contents were determined. Dry matter accumulation was faster in the beginning in the narrower spacing of $48 \mathrm{~cm}$, but in the end equal to the other densities; $N$ accumulation was highest in the narrower spacing, between 69 and 99 DAE. High plant density results in an earlier absorption peak of nutrients, suggesting an anticipated sidedressing with $N$ and $K$ in cotton grown in this system. For medium or highly fertile soils, the nutrient rates to be applied would not have to be changed at a higher plant density, because the amounts of N, P and K exported per $\mathrm{kg}$ in cotton fiber or cotton seed were not significantly different.
\end{abstract}

Index terms: Gossypium hirsutum, plant population, nutrient accumulation, cotton nutrition.

\section{INTRODUÇÃO}

O acúmulo de matéria seca pelo algodoeiro obedece a uma curva sigmoidal (Oosterhuis, 1990), de modo que a absorção dos nutrientes aumenta consideravelmente a partir dos 30 dias da emergência das plantas (DAE), a partir da emissão dos primeiros botões florais. A máxima absorção diária ocorre na fase de florescimento (Rosolem, 2001). O florescimento pleno corresponde não somente ao período de maior demanda por nutrientes, mas também ao período em que a taxa de crescimento radicular é máxima (Schwab et al., 2000). A redistribuição dos nutrientes móveis na planta ocorre a partir do início de desenvolvimento das maçãs, com o transporte de nutrientes das folhas para os tecidos reprodutivos. Esse fenômeno é mais evidente para o $\mathrm{K}$, e o fruto, especialmente as cápsulas, é o maior dreno de K (Rosolem \& Mikkelsen, 1989). O algodoeiro necessita de 48 a $85 \mathrm{~kg} \mathrm{ha}^{-1}$ de $\mathrm{N}$ para produzir uma tonelada de algodão em caroço, dependendo da variedade e das condições edafoclimáticas; em média, $43 \%$ desse total é exportado do campo (Rosolem et al., 2007). Entretanto, segundo Mullins \& Burmester (1990), a exportação de $\mathrm{N}$ via fibras e sementes pode chegar a $60 \%$ do $\mathrm{N}$ absorvido, estando $16 \%$ do total nos caules e aproximadamente $28 \%$ nas folhas; na parede da cápsula dos frutos encontram-se $14 \%$ e, nas sementes, aproximadamente $42 \%$ do total. Durante o desenvolvimento do fruto, este torna-se o principal dreno de $\mathrm{N}$ na planta e, consequentemente, ocorre a redistribuição do $\mathrm{N}$ das folhas e caules para os frutos (Rosolem \& Mikkelsen, 1989).
O algodoeiro absorve em média aproximadamente $12 \mathrm{~kg} \mathrm{ha}^{-1}$ de $\mathrm{P}$ por tonelada de algodão em caroço produzido, dos quais aproximadamente a metade é exportada (Carvalho et al., 2007). O P encontrase assim distribuído em plantas maduras de algodão: $12 \%$ nas hastes, $20 \%$ nas folhas, $16 \%$ nas cápsulas dos frutos e $53 \%$ nas sementes (Mullins \& Burmester, 1991). O pico diário de absorção de $\mathrm{P}$ (do início até a metade do florescimento) varia de 0,17 a $0,72 \mathrm{~kg} \mathrm{ha}^{-1}$ dia $^{-1}$ de $\mathrm{P}$; cerca de 21 a $36 \%$ do total é acumulado durante as duas primeiras semanas do florescimento (Furlani Jr. et al., 2001).

A exigência do algodoeiro em $\mathrm{K}$ é, em média, de aproximadamente $60 \mathrm{~kg} \mathrm{ha}^{-1}$ de K para cada tonelada de algodão em sementes, e a época de máxima taxa de absorção coincide com o florescimento (Furlani Jr. et al., 2001). Desse total, aproximadamente $18 \mathrm{~kg} \mathrm{ha}^{-1}$ são exportados (Carvalho et al., 2007). Durante o desenvolvimento dos frutos a absorção de K é muito pequena, mas a translocação para os frutos é intensa (Rosolem \& Mikkelsen, 1989), de modo que há muito $\mathrm{K}$ nas cápsulas dos frutos.

O cultivo adensado potencialmente encurta o ciclo, comparado ao sistema convencional, uma vez que o número final de frutos por planta não é superior a cinco ou seis, o que diminui o período de florescimento. Em espaçamentos mais estreitos a produção de frutos por unidade de área foliar é maior (Best et al., 1997), porém a produção total de matéria seca pode ser alterada ou não, dependendo das condições de cultivo (Jost \& Cothren, 2000; Silva et al., 2006). Assim, a hipótese do presente trabalho foi de que a redução do período de florescimento 
no sistema de cultivo adensado do algodoeiro pode alterar o acúmulo de matéria seca da planta e, consequentemente, o acúmulo de nutrientes.

$\mathrm{O}$ presente trabalho teve por objetivo avaliar a marcha de absorção dos nutrientes, N, P e K, possibilitando identificar a época de maior absorção e a quantidade desses nutrientes absorvida pelo algodoeiro, conduzido em sistemas adensado, intermediário e convencional.

\section{MATERIAL E MÉTODOS}

O experimento foi conduzido na Fazenda Santa Tereza, Paranapanema, SP, em lavoura comercial de algodão irrigado, no ano agrícola de 2009/2010, em um Latossolo Vermelho-Amarelo distrófico de textura muito argilosa (538 $\mathrm{g} \mathrm{kg}^{-1}$ de argila, $186 \mathrm{~g} \mathrm{~kg}^{-1}$ de silte e $276 \mathrm{~g} \mathrm{~kg}^{-1}$ de areia). A área foi cultivada anteriormente com feijoeiro. No dia 16/11/2009 a área foi dessecada com glyphosate $\left(2,7 \mathrm{~L} \mathrm{ha}^{-1}\right)+$ carfentrazone $\left(64 \mathrm{~mL} \mathrm{ha}^{-1}\right)$. A adubação mineral na semeadura constou da aplicação de $400 \mathrm{~kg} \mathrm{ha}^{-1}$ do formulado 08-28-12 + B e Zn (32 kg ha-1 de N, $112 \mathrm{~kg} \mathrm{ha}^{-1}$ de $\mathrm{P}_{2} \mathrm{O}_{5}$ e $48 \mathrm{~kg} \mathrm{ha}^{-1}$ de $\mathrm{K}_{2} \mathrm{O}$ ), baseando-se nos resultados da análise química do solo (Quadro 1). A semeadura foi realizada em 25 de novembro de 2009 de forma direta, sem o revolvimento do solo, utilizando-se a cultivar FM 966 LL. A emergência ocorreu nove dias após o plantio. A adubação de cobertura foi realizada com N (55 kg ha-1 aos 10 DAE e $45 \mathrm{~kg} \mathrm{ha}^{-1}$ aos $\left.24 \mathrm{DAE}\right)$ e K (91 kg ha-1 aos 29 DAE).

$\mathrm{O}$ manejo de herbicidas, inseticidas e fungicidas foi realizado conforme a necessidade e seguiu o padrão de aplicação da lavoura comercial. O manejo de reguladores de crescimento foi realizado buscando-se uma altura final da planta de $1,30 \mathrm{~m}$, utilizandose das moléculas cloreto de mepiquat e cloreto de clormequat. A desfolha foi feita aos $146 \mathrm{DAE}$, com a aplicação de thidiazuron. A aplicação do maturador ethephon + cyclanilidae ocorreu aos 152 DAE.

Os espaçamentos foram de 0,48, 0,75 e 0,96 m entre linhas, com estande final de 9,9 plantas $\mathrm{m}^{-1}$ (205.833 plantas $\left.\mathrm{ha}^{-1}\right), 10$ plantas $\mathrm{m}^{-1}$
(133.066 plantas $\mathrm{ha}^{-1}$ ) e 9,9 plantas $\mathrm{m}^{-1}$ (103.958 plantas ha-1), respectivamente. Três plantas competitivas (em linha e em sequência) por parcela foram colhidas aos 46, 69, 99, 139, 148 e 166 dias após a emergência (DAE) do algodoeiro. Após serem coletadas, as plantas foram separadas, conforme a época, em caule, folhas e frutos (botões florais, flores, maçãs, capulhos, fibra e semente). As amostras foram colocadas em estufa de circulação de ar forçada, à temperatura de $65^{\circ} \mathrm{C}$ durante $72 \mathrm{~h}$, para determinação da massa da matéria seca, sendo então moídas para posterior análise química de $\mathrm{N}$, $\mathrm{P}$ e K, utilizando-se a digestão sulfúrica para $\mathrm{N}$ e a nitroperclórica para $\mathrm{P} \mathrm{e} \mathrm{K}$, conforme método relatado por Malavolta et al. (1997). De posse dos resultados de massa seca de cada estrutura vegetativa e reprodutiva e dos teores de nutrientes de cada órgão em cada época amostrada, foram construídas curvas da marcha de absorção de N, P e K da planta inteira (soma de todos os órgãos).

O delineamento experimental foi o de blocos casualizados, com quatro repetições. As parcelas tinham área de $18 \mathrm{~m}^{2}(6 \times 3 \mathrm{~m})$. O estudo estatístico constou de análises de variância e de regressão, com ajuste de equações que apresentaram níveis de significância de no mínimo $5 \%$ de probabilidade pelo teste $\mathrm{F}$, sendo escolhidas as de maior coeficiente de determinação $\left(\mathrm{R}^{2}\right)$ com significado biológico. As médias dos tratamentos em cada época de amostragem foram comparadas pelo teste $t$ (LSD), conforme Banzato \& Kronka (2006).

\section{RESULTADOS E DISCUSSÃO}

$\mathrm{O}$ acúmulo de matéria seca (MS) na planta (caule+folha+fruto) seguiu o mesmo padrão em todas as densidades populacionais, não havendo diferença entre os tratamentos no acúmulo de MS por ocasião da colheita. No entanto, durante boa parte da fase de crescimento do algodoeiro, principalmente dos 69 aos 99 DAE, o acúmulo de MS foi maior no espaçamento de $48 \mathrm{~cm}$ (Figura 1a). Esse resultado foi consequência da maior produção de MS no caule (Figura 1b), uma vez que não houve diferença entre

Quadro 1. Características químicas do solo nas profundidades de 0-20 e 20-40 cm da área experimental

\begin{tabular}{|c|c|c|c|c|c|c|c|c|c|c|c|c|c|c|c|c|c|c|c|}
\hline Prof. & MO & $\underset{\mathrm{CaCl}_{2}}{\mathrm{pH}}$ & $\mathbf{P}_{\text {(resina) }}$ & $\mathbf{K}$ & $\mathbf{C a}$ & Mg & $\mathrm{Na}$ & $\mathrm{Al}$ & $\mathbf{H}$ & $\mathbf{H}+\mathbf{A l}$ & SB & CTC & V & $\mathrm{S}$ & B & $\mathbf{C u}$ & $\mathbf{F e}$ & Mn & $\mathrm{Zn}$ \\
\hline & $\mathrm{g} \mathrm{dm}^{-3}$ & & $\mathrm{mg} \mathrm{dm}{ }^{-3}$ & & & 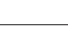 & \multicolumn{3}{|c|}{$-\mathrm{mmol}_{\mathrm{c}} \mathrm{dm}^{-3}$} & & & - & $\%$ & 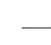 & 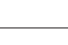 & \multicolumn{2}{|c|}{$\mathrm{mg} \mathrm{dm} \mathrm{m}^{-3}$} & 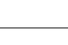 & - \\
\hline $0-20$ & 38,8 & 5,78 & 95 & 5,7 & 52 & 21 & 0,25 & 1 & 29 & 30 & 79 & 109 & 72 & 11 & 0,84 & 2,2 & 26 & 7 & 5,8 \\
\hline $20-40$ & 35,5 & 5,67 & 80 & 4,6 & 48 & 23 & - & 1 & 32 & 33 & 76 & 109 & 68 & - & - & - & - & - & - \\
\hline
\end{tabular}



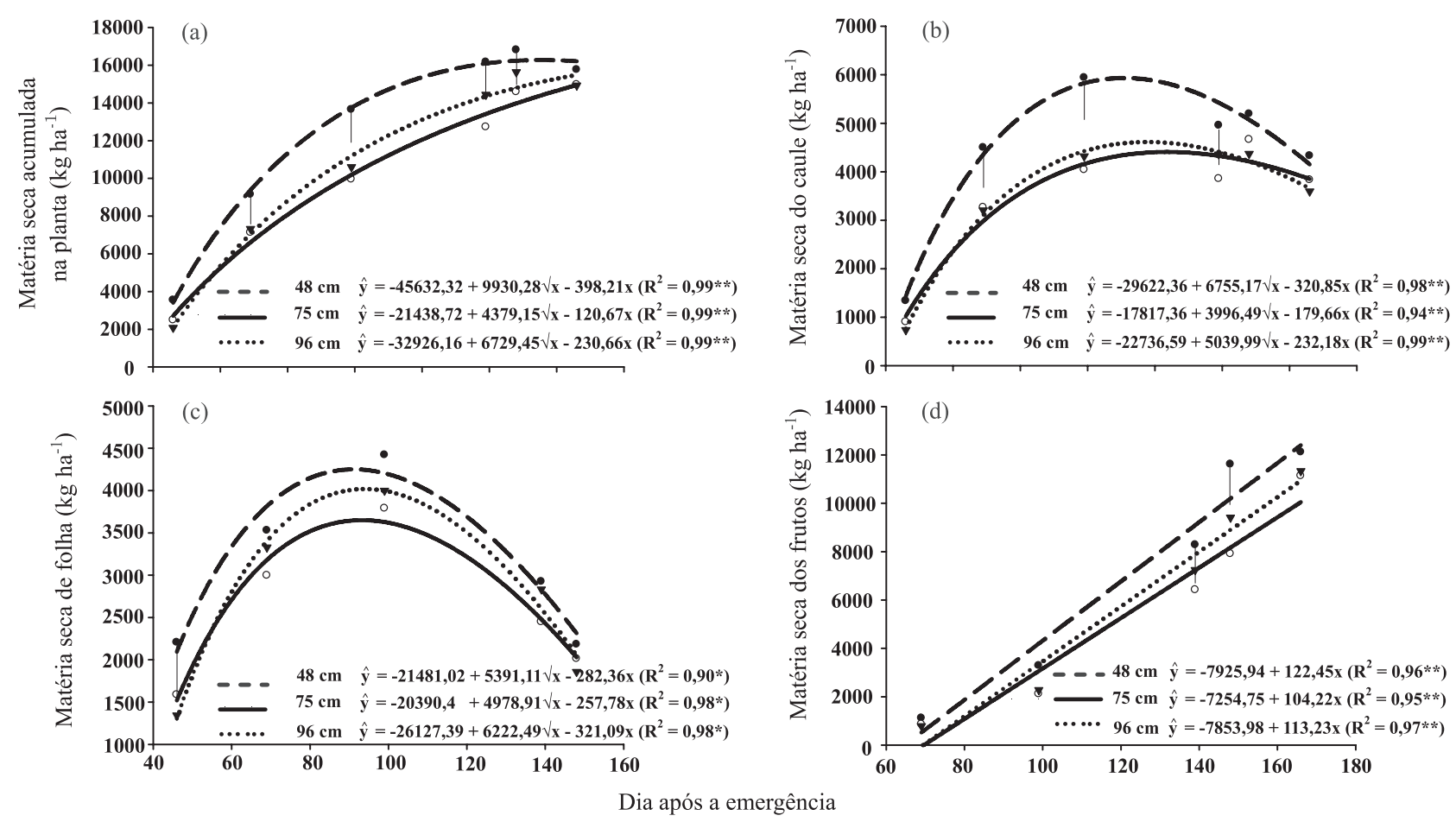

Figura 1. Acúmulo de matéria seca da planta (a), do caule (b), das folhas (c) e dos frutos (d) do algodoeiro cultivado nos espaçamentos de 48 (•), 75 ( ( ) e $96 \mathrm{~cm}$ ( $v$ ). A barra vertical representa a diferença mínima significativa $(\mathrm{dms} p<0,05)$.

os tratamentos nessas épocas para folhas (Figura 1c) e frutos (Figura 1d). Nas épocas mais tardias, após 130 DAE, além da produção de matéria seca de caule, também a produção de matéria seca de frutos foi maior no menor espaçamento. Resultados similares foram observados por Bozbek et al. (2006), quando as maiores densidades de semeadura resultaram em maior acúmulo de matéria seca em menor espaço de tempo. Kerby et al. (1990) verificaram que a maior densidade de semeadura (15 plantas $\mathrm{m}^{-2}$ ) produziu cerca de $8 \%$ a mais de matéria seca que a menor densidade ( 5 plantas $\mathrm{m}^{-2}$ ), e esse maior acúmulo ocorreu nas folhas e no caule, mas não nos frutos. Já no presente trabalho, observou-se que a diferença deveu-se ao aumento do acúmulo de matéria seca no caule e nos frutos. Por outro lado, apesar de nas maiores densidades populacionais haver maior quantidade de matéria seca e maior índice de área foliar, a partição de matéria seca para o desenvolvimento reprodutivo foi a mesma ou menor que em densidades populacionais menores (Darawsheh et al., 2009). Esse comportamento também foi observado neste trabalho, uma vez que não houve alteração na matéria seca de frutos acumulada por ocasião da colheita (Figura 1d). Apesar de Silva et al. (2006) terem observado maior produção biológica em espaçamentos mais largos, Jost \& Cothren (2000) não encontraram diferença significativa entre espaçamentos variando de 38 a $102 \mathrm{~cm}$, o que concorda com o presente trabalho.
Dessa forma, confirma-se que, em espaçamentos mais adensados, o acúmulo de matéria seca pelo algodoeiro é mais rápido, porém a quantidade final acumulada não é alterada. Isso ocorre porque, com espaçamentos menores, a intercepção de luz e a fotossíntese aumentam linearmente, devido ao fechamento precoce do dossel (Wells, 2006).

O menor espaçamento proporcionou maior acúmulo de $\mathrm{N}$ na planta, na mesma época em que a produção de matéria seca foi maior que nos outros espaçamentos (Figura 2a), principalmente devido ao maior acúmulo de $\mathrm{N}$ no caule (69 e 99 DAE Figura 2b), na folha (69 DAE - Figura 2c) e no fruto (99 DAE-Figura 2d). Observou-se também maior acúmulo de $\mathrm{N}$ nas folhas de algodão cultivado no espaçamento de $48 \mathrm{~cm}$ aos $46 \mathrm{DAE}$ (Figura 2c). Os resultados obtidos no espaçamento de $96 \mathrm{~cm}$ foram similares aos obtidos por Furlani Jr. et al. (2001) em espaçamento convencional. Assim, o maior acúmulo de N na planta parece ser uma consequência da maior produção de matéria seca, e não um efeito sobre a concentração do nutriente nos tecidos. O máximo acúmulo de $\mathrm{N}$ na planta ocorreu aos 113 , 138 e 126 dias após a emergência nos espaçamentos de 48, 75 e $96 \mathrm{~cm}$, respectivamente (Figura 2a), demonstrando que a redução dos espaçamentos, resultando em maior população, antecipou o período de maior acúmulo de $\mathrm{N}$ pela cultura. Contudo, as plantas cultivadas no espaçamento de $96 \mathrm{~cm}$ 

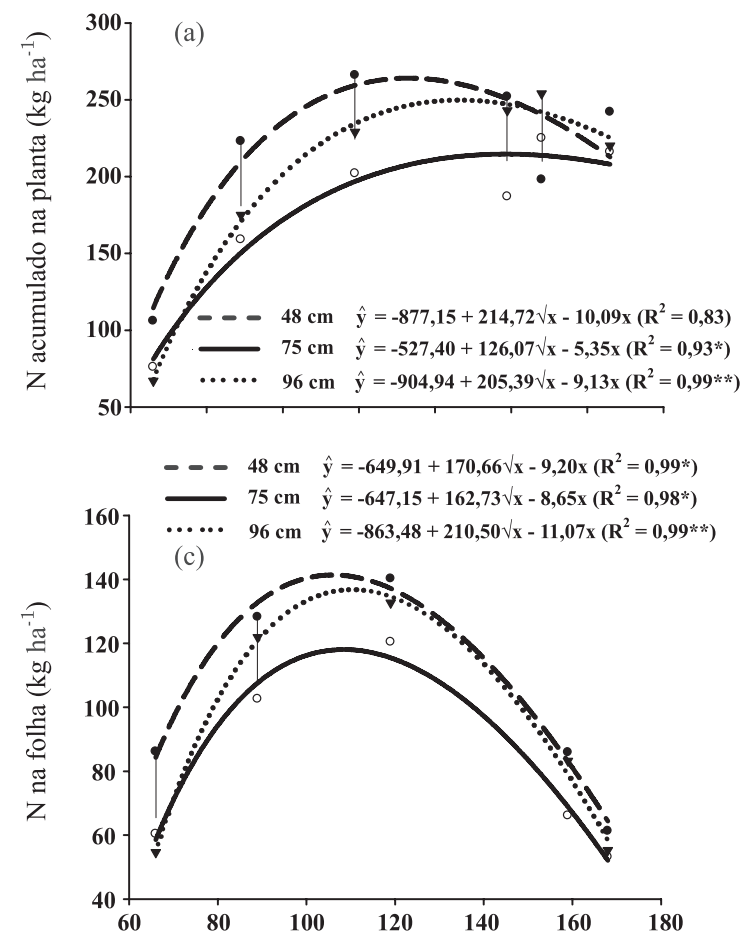
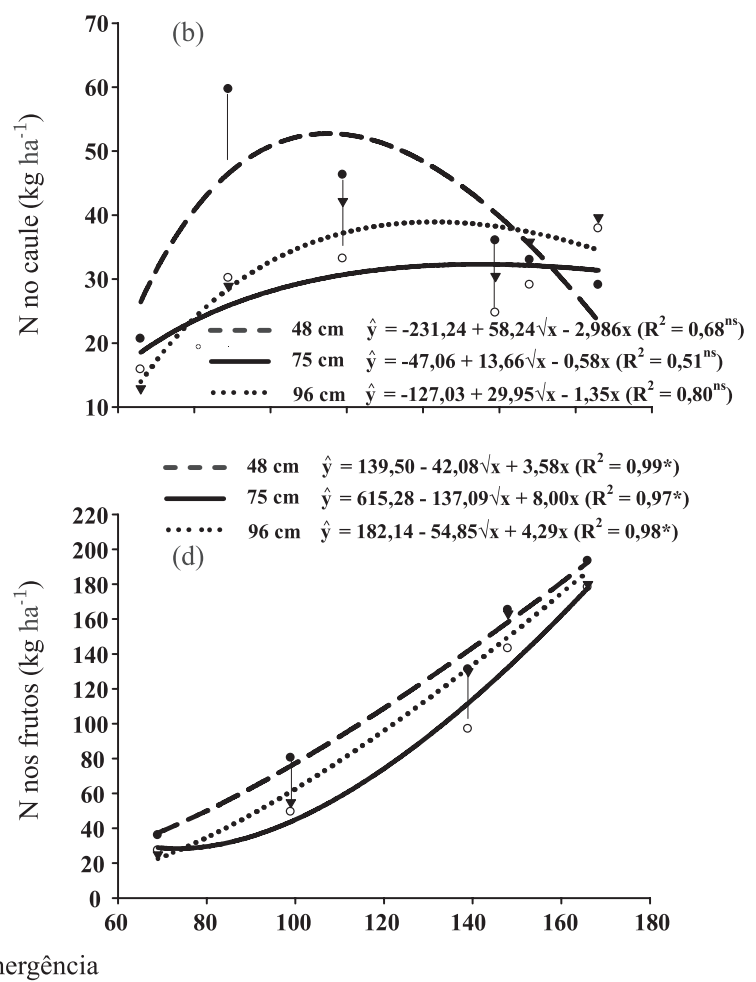

Figura 2. Nitrogênio acumulado na planta (a), no caule (b), nas folhas (c) e nos frutos (d) do algodoeiro cultivado nos espaçamentos de 48 (๑), 75 (०) e $96 \mathrm{~cm}(\mathrm{v})$. A barra vertical representa a diferença mínima significativa $(\mathrm{dms} p<0,05)$.

absorveram mais $\mathrm{N}$ em menos tempo que aquelas cultivadas no espaçamento de $75 \mathrm{~cm}$. Isso ocorreu porque houve maior produção de órgãos vegetativos (caule e folhas) no espaçamento mais largo.

A maior produção de matéria seca de folha (Figura 1c) e de caule (Figura 1b) também resultou em maior acúmulo de $\mathrm{N}$ nesses órgãos (Figura 2b,c). A época de maior absorção de $\mathrm{N}$ pela planta (Figura 2a) no espaçamento de $48 \mathrm{~cm}$ foi antecipada em razão da maior necessidade de $\mathrm{N}$ para formação e desenvolvimento de estruturas vegetativas (caule e folha), todavia o acúmulo total de $\mathrm{N}$ na planta por ocasião da colheita não foi alterado em resposta ao adensamento de plantas. Como consequência da alteração do período de florescimento e frutificação, o acúmulo de $\mathrm{N}$ nos frutos aos $99 \mathrm{DAE}$ foi maior quando o algodoeiro foi cultivado no espaçamento de $48 \mathrm{~cm}$, em relação aos demais espaçamentos.

Na época subsequente, aos 139 DAE, o algodoeiro cultivado nos espaçamentos de 48 e $96 \mathrm{~cm}$ acumulou maior quantidade de $\mathrm{N}$ nos frutos que no espaçamento de $75 \mathrm{~cm}$, uma vez que no espaçamento mais largo o período de florescimento horizontal é mais pronunciado que no de $75 \mathrm{~cm}$, devido ao maior crescimento dos ramos laterais e à formação de novas posições frutíferas. Assim, ocorre melhor partição de assimilados, proporcionando maior peso individual de frutos, visto que a quantidade final de $\mathrm{N}$ acumulado nos frutos não é alterada (Figura 2d).
De acordo com Dong et al. (2010), quando o algodoeiro foi cultivado em solos menos férteis e em maiores densidades de semeadura (7,5 plantas $\left.\mathrm{m}^{-2}\right)$, houve necessidade de adubação com N e K para suportar uma alta produção, sugerindo assim que a absorção de nutrientes também foi maior. Contudo, quando foi cultivado em solos de alta fertilidade, a produção foi independente da densidade de semeadura $\left(4,5\right.$ ou 7,5 plantas $\left.\mathrm{m}^{-2}\right)$, mas dependente da adubação com K. De acordo com o quadro 1, o solo onde as plantas foram cultivadas no presente experimento era bastante fértil (Silva \& Raij, 1996), e de fato não foram observadas diferenças entre as densidades de semeadura na produtividade final (Quadro 2).

O algodoeiro cultivado no espaçamento de $48 \mathrm{~cm}$ acumulou maior quantidade de $\mathrm{P}$ na planta que os demais espaçamentos aos 99 DAE (Figura 3a), uma vez que nessa época houve maior acúmulo de $\mathrm{P}$ em todos os órgãos da planta (Figura 3b,c,d). Também houve maior acúmulo de $\mathrm{P}$ no menor espaçamento $(48 \mathrm{~cm})$ aos 46 DAE para caule (Figura $3 \mathrm{~b}$ ) e folhas (Figura 3c) e, aos 69 DAE, apenas para o caule, sendo maior o acúmulo de $\mathrm{P}$ no algodoeiro cultivado em $48 \mathrm{~cm}$.

$\mathrm{O}$ pico de acúmulo de $\mathrm{P}$ na planta ocorreu aos 128, 160 e 136 DAE nos espaçamentos de 48, 75 e $96 \mathrm{~cm}$, com valores de 48,44 e $42 \mathrm{~kg} \mathrm{ha}^{-1}$ de $\mathrm{P}$, respectivamente. Novamente, o maior acúmulo de $\mathrm{P}$ em caule e em folhas foi determinante para 
Quadro 2. Nitrogênio, fósforo e potássio acumulado na fibra, semente e fibra+semente do algodoeiro cultivado nos espaçamentos de 48, 75 e $96 \mathrm{~cm}$ entre linhas

\begin{tabular}{|c|c|c|c|c|c|c|c|c|c|}
\hline & \multicolumn{3}{|c|}{$\mathrm{N}$ acumulado $\left(\mathrm{kg} \mathrm{Mg}^{-1}\right)^{\mathrm{ns}}$} & \multicolumn{3}{|c|}{$P$ acumulado $\left(\mathrm{kg} \mathrm{Mg}^{-1}\right)^{\mathrm{ns}}$} & \multicolumn{3}{|c|}{$\mathrm{K}$ acumulado $\left(\mathrm{kg} \mathrm{Mg}^{-1}\right)^{\mathrm{ns}}$} \\
\hline & \multicolumn{9}{|c|}{ Espaçamento $(\mathrm{cm})$} \\
\hline & 48 & 75 & 96 & 48 & 75 & 96 & 48 & 75 & 96 \\
\hline Fibra & 11,70 & 10,63 & 15,27 & 3,95 & 3,23 & 3,20 & 39,69 & 30,99 & 36,22 \\
\hline Semente & 45,69 & 39,85 & 47,14 & 9,05 & 7,66 & 9,05 & 13,71 & 11,48 & 15,05 \\
\hline Fibra+Semente & 33,82 & 29,78 & 35,06 & 7,27 & 6,13 & 6,83 & 22,78 & 18,18 & 23,07 \\
\hline
\end{tabular}

Ns: não significativo pelo teste t (LSD). Produtividade de fibra e rendimento em fibra (\%): $48 \mathrm{~cm}: 1591 \mathrm{~kg} \mathrm{ha}{ }^{-1}(34,92) ; 75 \mathrm{~cm}$ : $1.705 \mathrm{~kg} \mathrm{ha}^{-1}(34,44)$; e $96 \mathrm{~cm}: 1.598 \mathrm{~kg} \mathrm{ha}^{-1}(37,91)$.

modificar a marcha de absorção de $\mathrm{P}$ no espaçamento de $48 \mathrm{~cm}$ (Figura 3a,b,c). O acúmulo médio final de P na planta foi de $46 \mathrm{~kg} \mathrm{ha}^{-1}$ (51, 45 e $42 \mathrm{~kg} \mathrm{ha}^{-1}$ nos espaçamentos de 48, 75 e $96 \mathrm{~cm}$, respectivamente), valor este superior ao obtido por Rochester (2007), cujo acúmulo de $\mathrm{P}$ na planta de algodão cultivado no espaçamento de $1 \mathrm{~m}$ foi de $27 \mathrm{~kg} \mathrm{ha}^{-1}$ (variando de 18 a $43 \mathrm{~kg} \mathrm{ha}^{-1}$ ). Essas diferenças podem ser advindas das diferentes cultivares utilizadas, uma vez que cultivares mais modernas são mais exigentes em nutrientes.

$\mathrm{O}$ algodoeiro cultivado nos espaçamentos de $48 \mathrm{e}$ $96 \mathrm{~cm}$ acumulou maior quantidade de $\mathrm{K}$ na planta aos 69 DAE (Figura 4a), devido ao maior acúmulo desse nutriente no caule da planta (Figura $4 \mathrm{~b}$ ). Esse fato também foi observado na época posterior (99 DAE), em que o acúmulo de $\mathrm{K}$ na planta dos espaçamentos extremos (48 e $96 \mathrm{~cm}$ ) foi maior que no de $75 \mathrm{~cm}$; contudo, essa diferença ocorreu graças ao maior acúmulo de K no caule (Figura 4b), na folha (Figura 4c) e nos frutos (Figura 4d). Diferenças posteriores aos 99 DAE foram observadas em função do aumento da quantidade de $\mathrm{K}$ acumulada nos frutos (Figura 4d). Por não fazer parte de nenhum componente estrutural, o $\mathrm{K}$ possui alta mobilidade na planta, o que justifica a diminuição do seu acúmulo no caule e nas folhas (Figura 4b,c) e o aumento nos frutos (Figura 4d) - o principal dreno

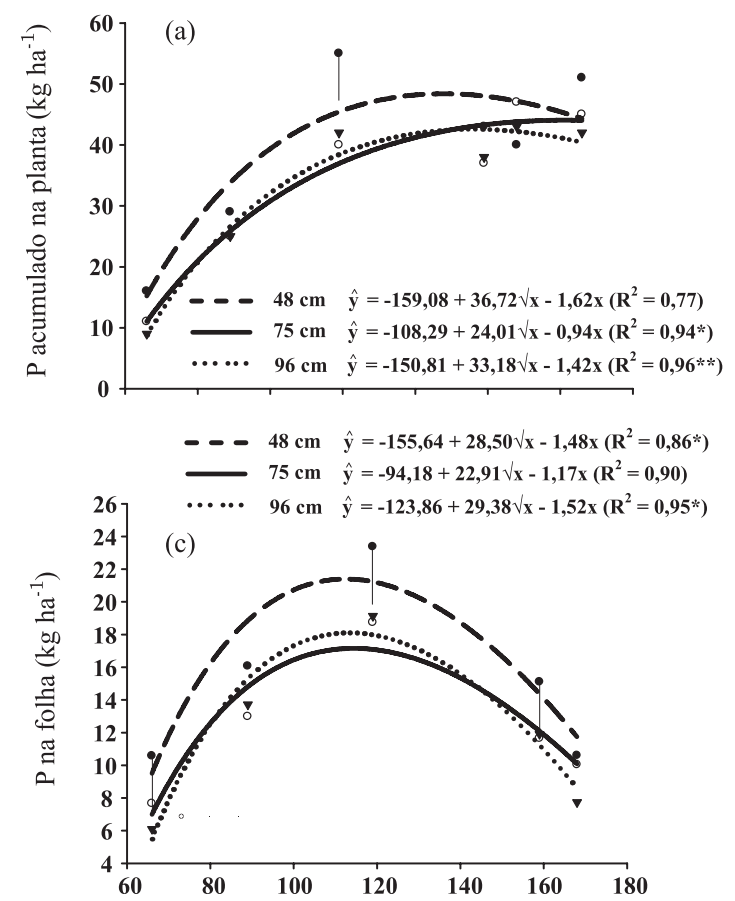

Dia após a emergência
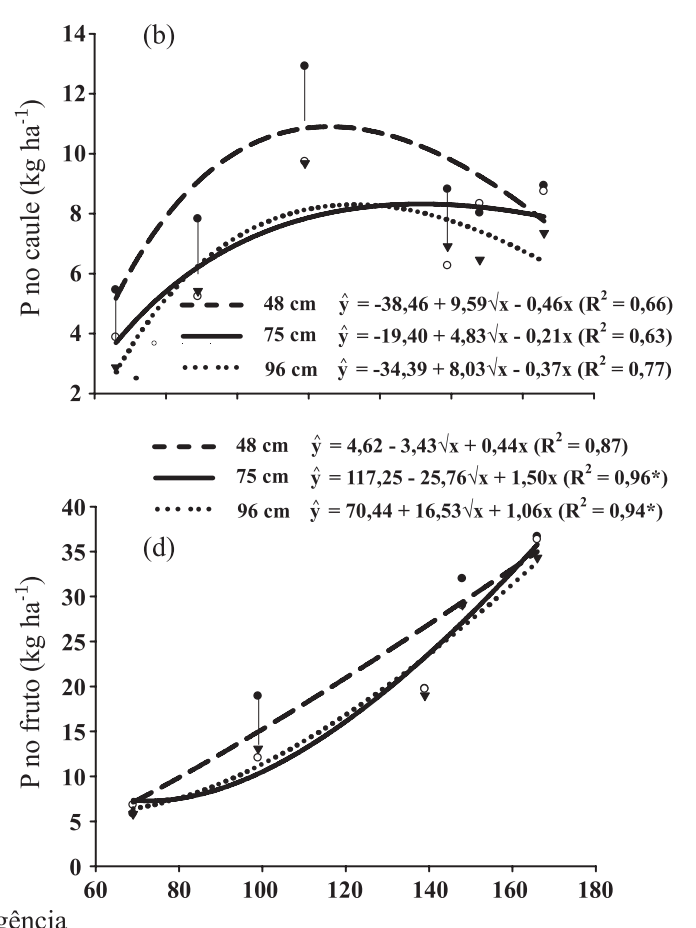

Figura 3. Fósforo acumulado na planta (a), no caule (b), nas folhas (c) e nos frutos (d) do algodoeiro cultivado nos espaçamentos de $48(\bullet), 75$ ( $\circ$ ) e $96 \mathrm{~cm}(\mathrm{v})$. A barra vertical representa a diferença mínima significativa (dms $p<0,05)$. 

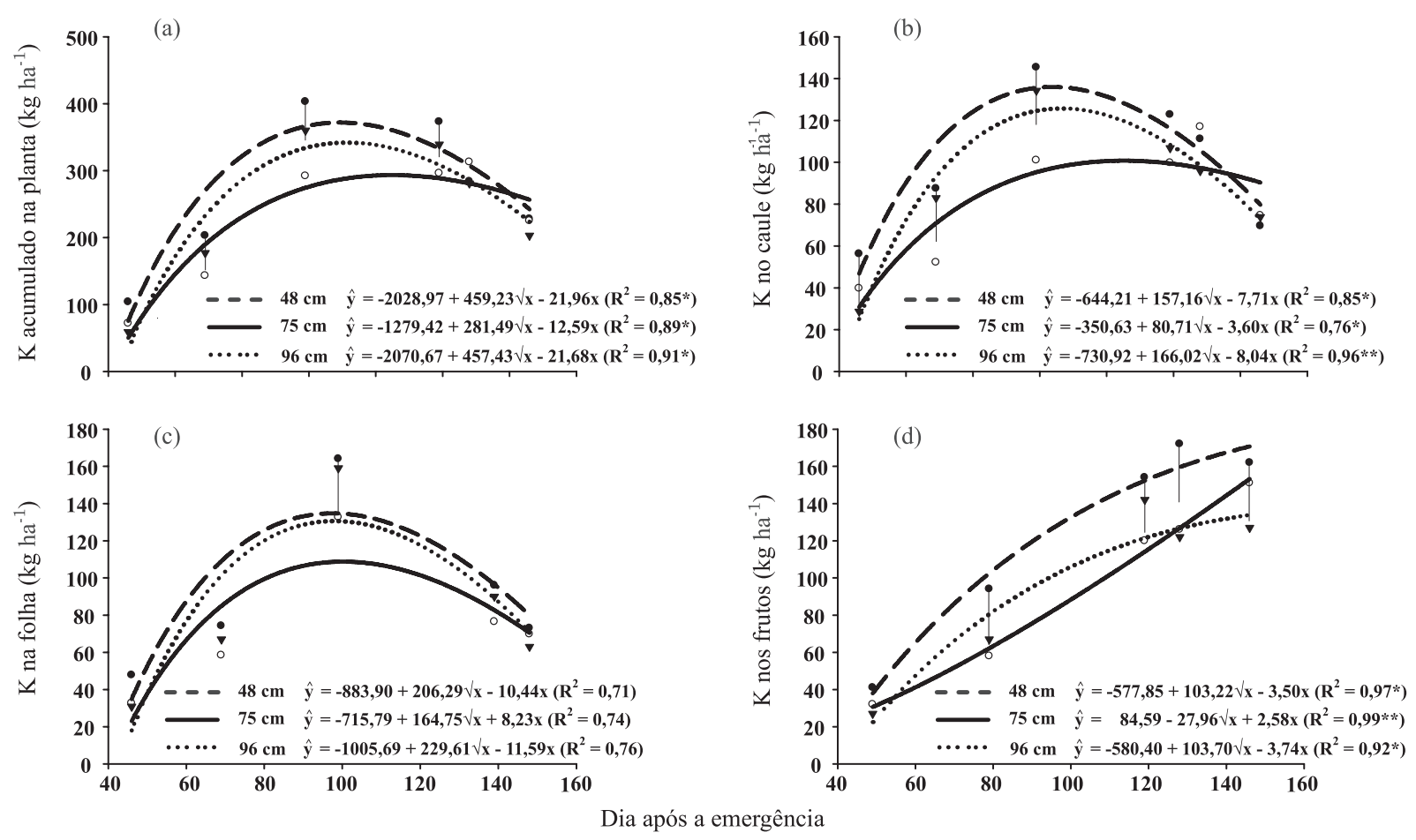

Figura 4. Potássio acumulado na planta (a), no caule (b), nas folhas (c) e nos frutos (d) do algodoeiro cultivado nos espaçamentos de 48 (•), 75 (०) e $96 \mathrm{~cm}(\mathrm{v})$. A barra vertical representa a diferença mínima significativa $(\mathrm{dms} \mathrm{p}<0,05)$.

na planta (Rosolem \& Mikkelsen, 1991). A época de maior demanda por K pelas plantas foi aos $110 \mathrm{DAE}$ para os espaçamentos de 48 e $96 \mathrm{~cm}$, coincidindo com o período de pleno florescimento e frutificação. Todavia, a época de maior necessidade de $\mathrm{K}$ pelo algodoeiro cultivado no espaçamento de $75 \mathrm{~cm}$ foi atrasada para os 124 DAE (Figura 4a), porque o acúmulo diário de matéria seca da parte vegetativa foi menor nesse espaçamento (Figura $5 \mathrm{~d}$ ), atrasando também a marcha de absorção do $\mathrm{K}$ nos frutos (Figura 5c). Uma possível explicação seria porque, neste arranjo, a distribuição espacial das plantas não estaria permitindo o máximo aproveitamento da luz incidente nessa época.

De acordo com Mendes (1960), a absorção de $\mathrm{K}$ pelo algodoeiro apresenta dois picos: o primeiro entre os 30 e 50 dias e o segundo aos 90 dias após a emergência, mas ambos situam-se no período do início ao pleno florescimento/desenvolvimento de frutos. Eaton \& Ergle (1957) observaram máxima absorção de K pelo algodoeiro até os 120 dias da emergência, a partir de quando houve diminuição do seu conteúdo na planta.

No início do desenvolvimemto, a taxa de acumulação diária de $\mathrm{N}$ nos órgãos vegetativos (caules+folhas) foi similar nos espaçamentos de 48 e $96 \mathrm{~cm}$ (Figura 5a). Após os $105 \mathrm{DAE}$, o acúmulo diário de $\mathrm{N}$ nos frutos das plantas cultivadas no espaçamento de $75 \mathrm{~cm}$ continuou aumentando até o final do ciclo - fato esse não observado nos espaçamentos de 48 e $96 \mathrm{~cm}$. As folhas representam a principal fonte de $\mathrm{N}$ aos frutos (Rosolem \& Mikkelsen, 1989), conforme foi observado no presente trabalho (Figura 2c); a partir dos 90-95 DAE, o acúmulo diário de $\mathrm{N}$ na parte vegetativa fica negativo, ocorrendo a translocação para os frutos.

A taxa de acúmulo de $\mathrm{P}$ nos órgãos vegetativos no início do desenvolvimento do algodoeiro foi maior quando cultivado nos espaçamentos extremos, apesar de a taxa ter se igualado aos 70 DAE nos três espaçamentos de cultivo. Para os frutos, o início do acúmulo de $\mathrm{P}$ foi antecipado nas plantas cultivadas no espaçamento de $48 \mathrm{~cm}$, porém, a partir dos 100 DAE até o final do ciclo, as taxas de acúmulo desse nutriente nos frutos foram idênticas nos espaçamentos de 48, 75 e $96 \mathrm{~cm}$ (Figura 5b). Em termos de manejo, a antecipação da absorção de $\mathrm{P}$ pelos frutos do algodoeiro cultivado no espaçamento de $48 \mathrm{~cm}$ não traz implicações, uma vez que o fornecimento do adubo fosfatado é feito por ocasião da semeadura da cultura ou em pré-plantio (Zancanaro, 2004).

No algodoeiro cultivado nos espaçamentos de 48 e $96 \mathrm{~cm}$, a taxa diária de acúmulo de $\mathrm{K}$ foi maior nos órgãos vegetativos no início do desenvolvimento, mas, a partir dos 90 DAE, não houve diferença entre os espaçamentos (Figura 5c). Quanto aos 

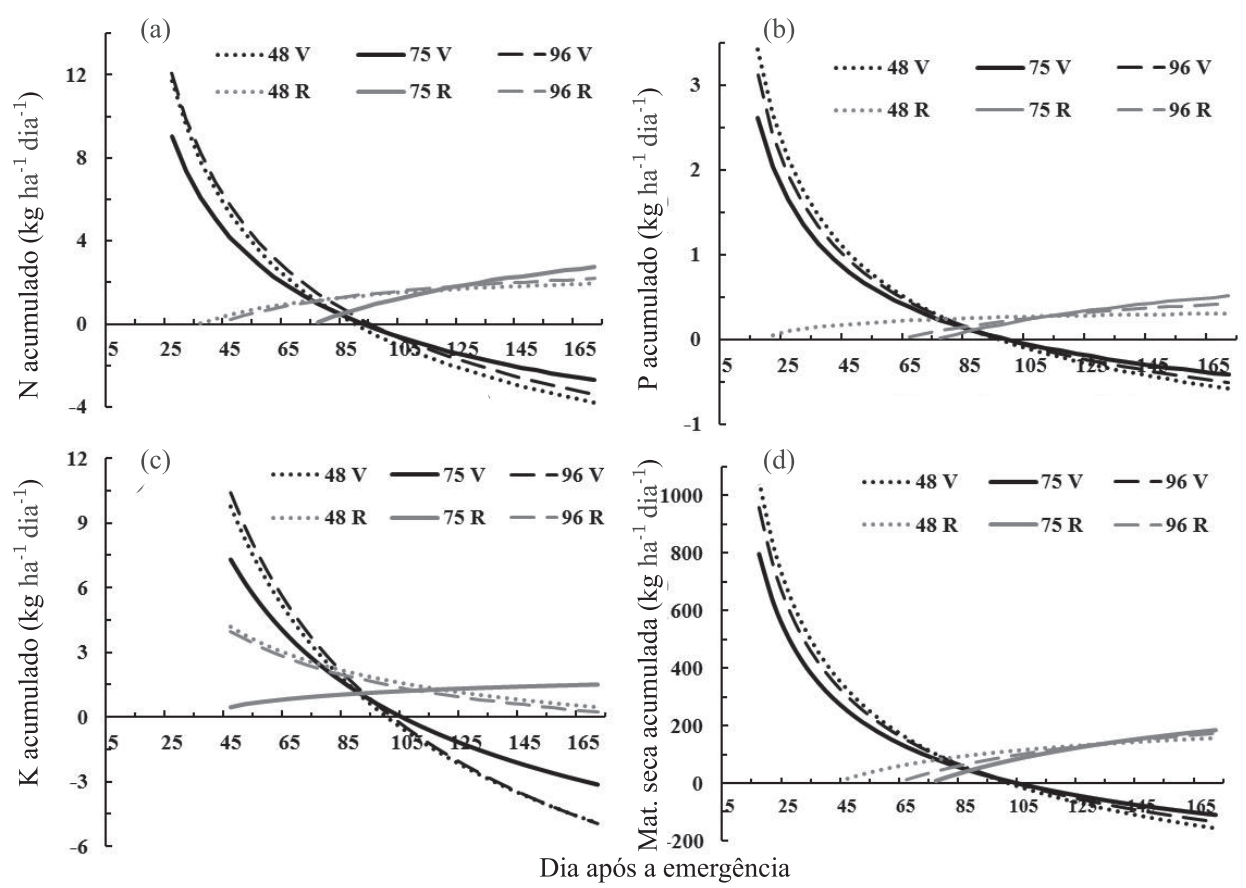

Figura 5. Taxa de acumulação diária de N (a), P (b), K (c) e matéria seca (d) nas partes vegetativas (V) e reprodutivas (R) do algodoeiro cultivado nos espaçamentos de 48, 75 e $96 \mathrm{~cm}$.

frutos, houve alta intensidade de acúmulo de $\mathrm{K}$ no algodoeiro cultivado nos espaçamentos de 48 e $96 \mathrm{~cm}$ no início do desenvolvimento; no entanto, no espaçamento de $75 \mathrm{~cm}$ esse comportamento foi diferenciado, com baixa taxa de acúmulo de $\mathrm{K}$ no início do desenvolvimento e posterior aumento e estabilização até o final do ciclo, enquanto nos demais espaçamentos a taxa de acúmulo diária diminuiu até o final do ciclo.

Não houve efeito dos espaçamentos de semeadura no acúmulo de $\mathrm{N}, \mathrm{P}$ e $\mathrm{K}$ na fibra e na semente (Quadro 2). De acordo com Dong et al. (2010), a quantidade de nutrientes exigida para produção de algodão em fibra está necessariamente relacionada com a quantidade produzida. Resultados extraídos de Rochester (2007) indicam que, conforme se aumenta a produção, proporcionalmente ocorre menor exportação de nutrientes como o N, P e $\mathrm{K}$, e isso também foi observado no presente trabalho (Quadro 2). A dose de $\mathrm{N}$ requerida para proporcionar a máxima produtividade biológica não variou em populações da ordem de 128 para 256 mil plantas ha-1 (Boquet, 2005), indicando que a quantidade absorvida não muda com o aumento da densidade populacional, como foi observado neste trabalho, embora a época de exigência tenha sido alterada (Figura 2a e Quadro 2).

Para a produção de uma tonelada de algodão em fibra, Halevy (1976) observou que a exportação de nutrientes foi, em média, de $60 \mathrm{~kg}$ de $\mathrm{N}, 11 \mathrm{~kg}$ de $\mathrm{P}$ e $26,5 \mathrm{~kg}$ de $\mathrm{K}$ - quantidades essas inferiores às observadas no presente trabalho (média de 91, 18,8 e
$60 \mathrm{~kg} \mathrm{ha}^{-1} \mathrm{de} \mathrm{N}, \mathrm{Pe} \mathrm{K}$, respectivamente). Uma possível explicação seria que as variedades mais modernas, oriundas de genótipos importados, mostram-se mais sensíveis à deficiência de nutrientes, especialmente o $\mathrm{K}$, e isso pode ocorrer por terem capacidade inferior de absorção de nutrientes do solo (Rosolem, 2001), podendo haver diferenças entre cultivares da ordem de 40, 43 e $21 \%$ na quantidade requerida de $\mathrm{N}, \mathrm{P}$ e $\mathrm{K}$, respectivamente, para produzir a mesma quantidade de algodão em fibra (Unruh \& Silvertooh, 1996). No entanto, comparando com os valores citados em Tessaro et al. (2006), em que foram empregados materiais genéticos mais modernos, proporcionalmente, a exportação de nutrientes no presente trabalho foi maior para $\mathrm{N} \mathrm{e} \mathrm{K}$ e similar para o $\mathrm{P}$, fato esse que também corrobora os resultados de Dorahy et al. (2004), que reportaram exportação de $15 \mathrm{~kg} \mathrm{ha}^{-1}$ de P. Isso pode ser advindo da disponibilidade de nutrientes no solo, já que, em condições de maior fertilidade do solo, a planta absorve mais nutrientes, e a partir de certo ponto isso não se reflete em aumento de produtividade, caracterizando o consumo de luxo (Duggan et al., 2009), o que pode ser observado no quadro 2 , cuja maior exportação de nutrientes não se refletiu em maior produtividade.

\section{CONCLUSÕES}

1. O adensamento antecipa o pico de absorção de nutrientes, sugerindo a antecipação das adubações 
de cobertura com $\mathrm{N}$ e $\mathrm{K}$ no algodoeiro cultivado nesse sistema.

2. Para condições de fertilidade média/alta, a dose de nutrientes a ser aplicada não precisaria ser alterada em função do aumento da densidade de plantas, pois não há variação na quantidade final acumulada nem nas quantidades de N, P e K exportadas por kg de algodão produzido, em fibra ou em caroço.

\section{AGRADECIMENTO}

À Fapesp, pela concessão da bolsa de iniciação científica ao terceiro autor.

\section{LITERATURA CITADA}

BANZATO, D.A. \& KRONKA, S.N. Experimentação agrícola. 4.ed. Jaboticabal, Funep, 2006. 237p.

BEST, E.C.; RINEY, J.B. \& KRIEG, D.R. Factors affecting source-sink relations in cotton. In: BELTWIDE COTTON CONFERENCES, Memphis, 1997. Proceedings... Memphis, National Cotton Council of America, 1997. v.2. p.1387-1389.

BOQUET, D.J. Cotton in ultra-narrow row spacing: Plant density and nitrogen fertilizer rates. Agron. J., 97:279$287,2005$.

BOZBEK, T.; VOLKAN, S. \& AYDIN, U. The effect of sowing date and plant density on cotton yield. J. Agron., 5:122-125, 2006.

CARVALHO, M.C.S.; FERREIRA, G.B. \& STAUT, L.A. Nutrição, calagem e adubação do algodoeiro. In: FREIRE, E., ed. Algodão no Cerrado do Brasil. Brasília, ABRAPA, 2007. p.581-647.

DARAWSHEH, M.K.; KHAH, E.M.; AIVALAKIS, G.; CHACHALIS, D. \& SALLAKU, F. Cotton row spacing and plant density cropping systems I. Effects on accumulation and partitioning of dry mass and LAI. J. Food Agric. Environ., 7:258-261, 2009 .

DONG, H.; KONG, X.; LI, W.; TANG, W. \& ZHANG, D. Effects of plant density and nitrogen and potassium fertilization on cotton yield and uptake of major nutrients in two fields with varying fertility. Field Crops Res., 119:106-113, 2010.

DORAHY, C.G.; ROCHESTER, I.J. \& BLAIR, G.J. Response of field-grown cotton (Gossypium hirsutum L.) to phosphorus fertilisation on alkaline soils in eastern Australia. Austr. J Soil Res., 42:913-920, 2004.

DUGGAN, B.L.; YEATES, S.J.; GAFF, N. \& CONSTABLE, G.A. Phosphorus fertilizer requirements and nutrient uptake of irrigated dry-season cotton grown on virgin soil in tropical Australia. Comm. Soil Sci. Plant Anal., 40:2616-2637, 2009.

EATON, F. M. \& ERGLE, D. R. Mineral nutrition of the cotton plant. Plant Phys., 32:169-175, 1957.
FURLANI JR., E.; SILVA, N.M.; BUZETTI, S.; SÁ, M.E.; ROSOLEM, C.A. \& CARVALHO, M.A.C. Extração de macronutrientes e acúmulo de massa seca de algodão cv. IAC 22. Cult. Agron., 10:71-87, 2001.

HALEVY, J. Growth rate and nutrient uptake of two cotton cutivars grown under irrigation. Agron. J., 68:701-705, 1976.

JOST, P.H. \& COTHREN, J.T. Growth and yield comparisons of cotton planted in conventional and ultra-narrow row spacings. Crop Sci., 40:430-435, 2000.

KERBY, T.A.; CASSMAN, K.G. \& KEELEY, M. Genotypes and plant densities for narrow-row cotton systems. ii. leaf area and dry-matter partitioning. Crop Sci., 30:649-653, 1990.

MALAVOLTA, E.A.; VITTI, G.C. \& OLIVEIRA, A.S. Avaliação do estado nutricional das plantas: princípios e aplicações. Piracicaba, Potafos, 1997. 201p.

MENDES, H.C. Nutrição do algodoeiro II - Absorção mineral por plantas cultivadas em soluções nutritivas. Bragantia, 19:435-457, 1960.

MULLINS, G.L. \& BURMESTER, C.H. Dry matter, nitrogen, phosphorus, and potassium accumulation by four cotton varieties. Agron. J., 82:729-736, 1990.

OOSTERHUIS, D.M. Growth and development of a cotton plant. In: MILEY, W.N. \& OOSTERHUIS, D.M., eds. Nitrogen nutrition of cotton: Practical issues. Madison, ASA/CSSA/ SSSA, 1990. p.1-24.

ROCHESTER, I.J. Nutrient uptake and export from an Australian cotton field. Nutr. Cycl. Agroec., 77:213-223, 2007.

ROSOLEM, C.A. Problemas em nutrição mineral, calagem e adubação do algodoeiro. Inf. Agron., 95:1-17, 2001. (Encarte Técnico)

ROSOLEM, C.A. \& MIKKELSEN, D.S. Nitrogen source-sink relationship in cotton. J. Plant Nutr., 12:1417-1448, 1989.

ROSOLEM, C.A. \& MIKKELSEN, D.S.. Potassium absorption and partitioning in cotton as affected by periods of potassium deficiency. J. Plant Nutr., 14:1001-1016, 1991.

ROSOLEM, C.A.; ZANCANARO, L.Z. \& TESSARO, L.C. Nitrogênio e enxofre na cultura do algodoeiro. In: YAMADA, T.; ABDALLA, S.R.S. \& VITTI, G.C. Nitrogênio e enxofre na agricultura brasileira. Piracicaba, IPNI, 2007. p.321-347.

SCHWAB, G.J.; MULLINS, G.L. \& BURMESTER, C.H. Growth and nutrient uptake by cotton roots under field conditions. Comm. Soil Sci. Plant. Anal., 31:149-164, 2000.

SILVA, A.V.; CHIAVEGATO, J.E.B.; CARVALHO, L.H. \& KU. BIAK, D.M. Crescimento e desenvolvimento do algodoeiro em diferentes configurações de semeadura. Bragantia, 65:407-411, 2006.

SILVA, N.M. \& RAIJ, B.van. Recomendações de adubação e calagem para o Estado de São Paulo. Campinas, Instituto Agronômico/Fundação IAC, 1996. p.107-111. (IAC. Boletim Técnico, 100) 
TESSARO, L.C.; ZANCANARO, L. \& HILLESHEM, J. Manejo da adubação com enxofre na cultura do algodão. Rondonópolis, 2006. (Relatório Técnico FACUAL)

UNRUH, B.L. \& SILVERTOOTH, J.C. Comparisons between an upland and a pima cotton cultivar: II. Nutrient uptake and partitioning. Agron. J., 88:589-595, 1996.
WELLS, R. Photosynthesis: The path to higher yield and quality. In: BELTWIDE COTTON CONFERENCE, 2006, San Antonio, Texas. Proceedings... Memphis, National Cotton Council of America, 2006. v.1. p.1762-1764.

ZANCANARO, L. Fósforo na cultura do algodão do Mato Grosso. In: YAMADA, T. \& ABDALLA, S.R.S., eds. SIMPÓSIO SOBRE FÓSFORO NA AGRICULTURA BRASILEIRA, Piracicaba, 2004. Anais... Piracicaba, Potafos, 2004. 726p. 